\title{
Value of quantitative dynamic contrast-enhanced and diffusion- weighted magnetic resonance imaging in predicting extramural venous invasion in locally advanced gastric cancer and prognostic significance
}

\author{
Yongjian Zhu'" ${ }^{1 \#}$ Yutao Zhou ${ }^{1 \#}$, Wen Zhang', Liyan Xue ${ }^{3}$, Ying $\mathrm{Li}^{1}$, Jun Jiang ${ }^{1}$, Yuxin Zhong ${ }^{4}$, Sicong Wang ${ }^{5}$, \\ Liming Jiang ${ }^{1}$ \\ ${ }^{1}$ Department of Imaging Diagnosis, National Cancer Center/National Clinical Research Center for Cancer/Cancer Hospital, Chinese Academy \\ of Medical Sciences and Peking Union Medical College, Beijing, China; ${ }^{2}$ Department of Medical Oncology, National Cancer Center/National \\ Clinical Research Center for Cancer/Cancer Hospital, Chinese Academy of Medical Sciences and Peking Union Medical College, Beijing, China; \\ ${ }^{3}$ Department of Pathology, National Cancer Center/National Clinical Research Center for Cancer/Cancer Hospital, Chinese Academy of Medical \\ Sciences and Peking Union Medical College, Beijing, China; ${ }^{4}$ Department of Pancreatic and Gastric Surgery, National Cancer Center/National \\ Clinical Research Center for Cancer/Cancer Hospital, Chinese Academy of Medical Sciences and Peking Union Medical College, Beijing, China; \\ ${ }^{5}$ GE Healthcare, Life Sciences, Beijing, China
}

\#These authors contributed equally to this work.

Correspondence to: Liming Jiang. Department of Imaging Diagnosis, National Cancer Center/National Clinical Research Center for Cancer/Cancer Hospital, Chinese Academy of Medical Sciences and Peking Union Medical College, 17 Panjiayuan South Road, Chaoyang District, Beijing 100021, China. Email: dr_jiangliming@126.com.

Background: Extramural venous invasion (EMVI) has been found to be related to poor prognosis in gastric cancer. Preoperative diagnosis of EMVI is challenging, as it can only be detected by surgical pathology. The present study aimed to investigate the value of quantitative dynamic contrast-enhanced magnetic resonance imaging (DCE-MRI) and diffusion-weighted imaging (DWI) in predicting EMVI preoperatively, and to determine the relationship between prediction results and prognosis in patients with locally advanced gastric cancer (LAGC).

Methods: Between January, 2015, and June, 2017, 79 LAGC patients underwent MRI preoperatively were enrolled in this study. Pathological EMVI (pEMVI) was used as the gold standard for diagnosis. The differences in quantitative DCE-MRI and DWI parameters between groups with different pEMVI status were analyzed. Multivariate logistic regression was used to build the combined prediction model for pEMVI with statistically significant quantitative parameters. The performance of the model for predicting pEMVI was evaluated using receiver operating characteristic (ROC) analysis. Patients were grouped based on MRI-predicted EMVI (mrEMVI). Kaplan-Meier analysis was used to investigate the relationship between mrEMVI and 2-year recurrence-free survival (RFS).

Results: Of the 79 LAGC patients who underwent MRI, 29 were pEMVI positive and 50 were pEMVI negative. Among the patients' clinical and pathological characteristics, only postoperative staging showed a significant difference between the 2 groups $(\mathrm{P}=0.015)$. The pEMVI-positive group had higher volume transfer constant $\left(\mathrm{K}^{\text {trans }}\right)$ and rate constant $\left(\mathrm{k}_{\mathrm{ep}}\right)$, and lower apparent diffusion coefficient (ADC) values than the negative group (0.189 vs. $0.082 \mathrm{~min}^{-1}, 0.687$ vs. $0.475 \mathrm{~min}^{-1}$, and $1.230 \times 10^{-3}$ vs. $1.463 \times 10^{-3} \mathrm{~mm}^{2} / \mathrm{s}$, respectively; $\mathrm{P}<0.05)$. Quantitative parameters, $\mathrm{K}^{\text {trans }}$ and $\mathrm{k}_{\mathrm{ep}}$, and $\mathrm{ADC}$ values, were independently associated with pEMVI which odds ratio values were 3.66, 2.65, and $0.30(\mathrm{P}<0.05)$, respectively, using multivariate logistic regression. ROC analysis showed that the area under the curve, sensitivity, specificity, positive predictive value, and negative predictive value in predicting $\mathrm{pEMVI}$ using combined $\mathrm{K}^{\text {trans }}, \mathrm{k}_{\mathrm{ep}}$, and ADC 
values were $0.879,72.4 \%, 96 \%, 91.3 \%$, and $85.7 \%$, respectively. A total of 23 cases were considered to be mrEMVI positive, and 56 cases were considered to be mrEMVI negative, according to the predictive results. The median RFS of the mrEMVI-positive group was significant lower than the negative group (21.7 vs. 31.2 months), and the 2-year RFS rate in the mrEMVI-positive group was significantly lower than that of the negative group ( $43.6 \%$ vs. $72.5 \%, \mathrm{P}=0.010)$.

Conclusions: The quantitative DCE-MRI parameters, $\mathrm{K}^{\text {trans }}$ and $\mathrm{k}_{\mathrm{ep}}$, and DWI parameter, ADC, are independent predictors of pEMVI in LAGC; mrEMVI was confirmed to be a poor prognostic predictor for RFS.

Keywords: Gastric cancer; blood vessels; contrast media; diffusion-weighted imaging; prognosis

Submitted Feb 12, 2020. Accepted for publication Sep 03, 2020.

doi: 10.21037/qims-20-246

View this article at: http://dx.doi.org/10.21037/qims-20-246

\section{Introduction}

Gastric cancer is the fifth most common cancer and the third leading cause of cancer-related mortality globally (1). The symptoms of early gastric cancer are vague; consequently, $32 \%$ of patients already have locally advanced gastric cancer (LAGC) at the time of diagnosis (2). Despite early diagnosis and the development of therapeutic modalities, the overall 5 -year survival rate is only approximately $26 \%$, due to a high frequency of postoperative recurrence, even after radical gastrectomy $(2,3)$.

Studies in recent years have shown that preoperative neoadjuvant therapy can control local lesions in LAGC patients, improve the radical resection rate, and subsequently prolong disease-free and overall survival (4). However, some studies have failed to find the benefit of neoadjuvant therapy (5); therefore, a consensus on neoadjuvant therapy for patients with gastric cancer has not been reached. These inconsistent results might be caused by the heterogeneity of LAGC. Depth of tumor invasion ( $\mathrm{T}$ stage) and lymph node status ( $\mathrm{N}$ stage) are important pathological factors that affect postoperative survival in LAGC, and are applied in the American Joint Committee on Cancer (AJCC)/International Union Against Cancer (UICC) staging system (6). Patients with similar clinicopathological factors have also been reported to have different survival outcomes (7). Therefore, other riskstratification factors affecting postoperative recurrence and survival are needed to guide clinical treatment.

Recently, extramural venous invasion (EMVI) has been found to be an independent risk factor for recurrence and distant metastasis in patients with gastric and rectal cancer $(8,9)$. EMVI is defined as the invasion of the veins beyond the muscularis propria of the gastrointestinal tract by tumor cells, which is considered to be a mode of tumor spread $(10,11)$. EMVI is an adverse prognostic factor in rectal cancer (8), and it has been included as an important imaging parameter prior to neoadjuvant therapy in the National Comprehensive Cancer Network guidelines for rectal cancer. However, few studies on EMVI imaging in gastric cancer have been conducted.

Quantitative dynamic contrast-enhanced magnetic resonance imaging (DCE-MRI) and diffusion-weighted imaging (DWI) are commonly used techniques in clinical practice (12). Quantitative DCE-MRI was demonstrated to non-invasively calculate hemodynamic parameters (13), such as tissue perfusion, microvascular permeability, and vascular cell clearance volume, through a contrast pharmacokinetic model. DWI reflects the diffusion movement of water molecules by calculating the apparent diffusion coefficient (ADC) of tumor tissue. Quantitative DCE-MRI parameters and ADC values have been reported to be closely related to clinical, histopathological, and prognostic factors of various tumors (14-17). However, due to respiratory movement and gastrointestinal motility, these functional MRI techniques are rarely used in gastric research. To the best of our knowledge, no studies have been performed on the prediction and prognostic impact of EMVI using quantitative DCE-MRI and DWI in gastric cancer.

The present study aimed to explore the predictive value of combined quantitative DCE-MRI and DWI on pathological EMVI (pEMVI), and to determine the relationship between prediction results and prognosis in terms of recurrence-free survival (RFS) in LAGC patients after radical gastrectomy and adjuvant chemotherapy in order to guide clinical decision-making. 


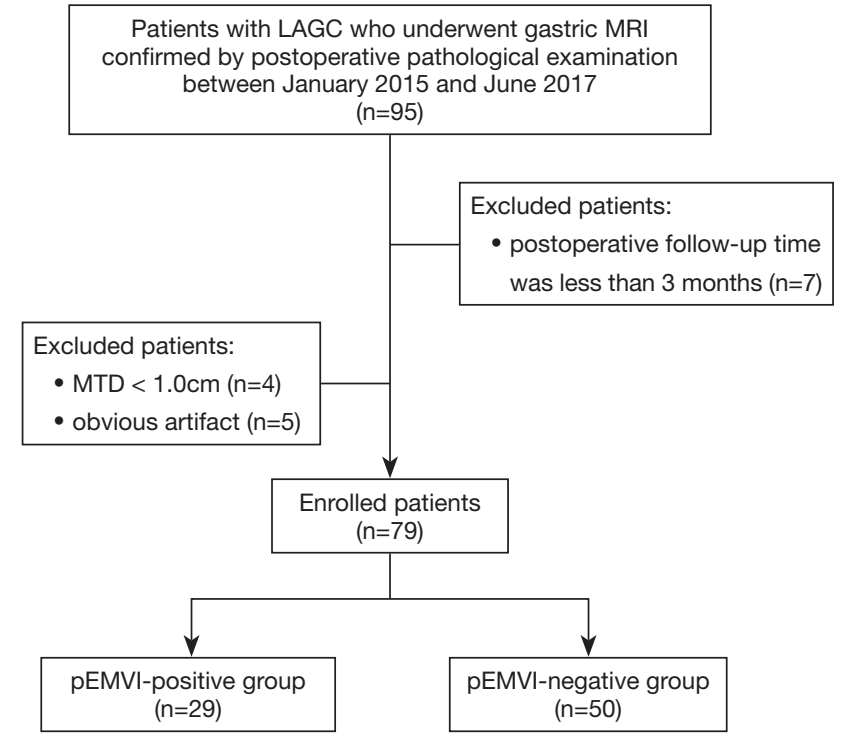

Figure 1 Recruitment of the study population and the exclusion criteria. LAGC, locally advanced gastric cancer; MRI, magnetic resonance imaging; MTD, maximum tumor diameter; pEMVI, pathological extramural venous invasion.

\section{Methods}

\section{Patients}

The Independent Ethics Committee of the Cancer Hospital, Chinese Academy of Medical Sciences (Beijing, China) approved this retrospective study and waived the requirement for informed patient consent. Between January, 2015, and June, 2017, 95 patients with newly diagnosed resectable LAGC undergoing gastric MRI preoperatively were recruited for participation in the present study. The inclusion criteria were as follows: (I) TNM stage cT2-4aN0-3M0, based on preoperative computed tomography (CT), MRI, or endoscopic ultrasonography (18); (II) gastric cancer and pEMVI status confirmed by surgical pathology; (III) R0 radical gastrectomy within 30 days after MRI; and (IV) regular postoperative follow-up. Sixteen patients were excluded for the following reasons: (I) obvious motion artifact related to respiration and bowel peristalsis during the acquisition ( $\mathrm{n}=5$ ); (II) maximum tumor diameter (MTD) $<1 \mathrm{~cm}(\mathrm{n}=4)$; and (III) postoperative follow-up time $<3$ months $(\mathrm{n}=7)$. Finally, 79 patients were enrolled in the present study, including 68 males and 11 females, with a median age of 58 years old (range, 30-70 years). Figure 1 shows the patient selection flowchart.

\section{MRI examination}

All patients were fasted for 6-8 h before MRI to empty the gastrointestinal tract. If no contraindications were present (i.e., glaucoma, prostate hypertrophy, asthma, or severe heart disease), $10 \mathrm{mg}$ anisodamine (Minsheng Pharmaceutical, China) was injected intramuscularly to prevent gastrointestinal motility, and $800-1,000 \mathrm{~mL}$ water was orally administered to dilate the stomach wall before MRI.

All of the magnetic resonance images were acquired using a 3.0 T body MRI system (Discovery MR750 3.0T; GE Medical Systems, USA) equipped with an 8-channel, phased-array body coil.

DWI was performed using spin-echo echo-planar imaging in the respiratory-gating mode in the transverse plane, with the following scanning parameters: b-values: 0 and $800 \mathrm{~s} / \mathrm{mm}^{2}$; repetition time (TR): $8,000 \mathrm{~ms}$; echo time (TE): $60 \mathrm{~ms}$; field of view (FOV): $38 \mathrm{~cm} \times 38 \mathrm{~cm}$; matrix: $160 \times 128$; section thickness: $5 \mathrm{~mm}$; and gap: $1 \mathrm{~mm}$. Quantitative DCE-MRI acquisitions were performed with breath-hold multiphase liver acceleration volume acquisition sequence. According to a recent study (19), pre-contrast T1 mapping with multiflip angles $\left(3^{\circ}, 6^{\circ}, 9^{\circ}\right.$, and $12^{\circ}$ ) was acquired in the axial plane before dynamic scanning (TR: $2.6 \mathrm{~ms}$, TE: $1.2 \mathrm{~ms}$, FOV: $38 \mathrm{~cm} \times 38 \mathrm{~cm}$, matrix: $224 \times 224$, section thickness: $5 \mathrm{~mm}$, gap: $0 \mathrm{~mm}$ ). A bolus of gadopentetate dimeglumine (Magnevist, Bayer Schering, Germany) at a constant dose of $0.1 \mathrm{mmol} / \mathrm{kg}$ was power injected, followed by a $20 \mathrm{~mL}$ saline flush at a rate of $2.5 \mathrm{~mL} / \mathrm{s}$ for all patients. The scanning parameters and range of dynamic sequences were the same as those for $\mathrm{T} 1$ mapping, with a flip angle of $15^{\circ}$. Forty-two repetitions were acquired at a temporal resolution of $6 \mathrm{~s}$, with an interval of $5-10 \mathrm{~s}$ for each of the three consecutive phases; the total scanning time for DCE-MRI was 5-6 min.

\section{MRI analysis}

The MTD was measured at the largest section of the tumor using good image quality. The DCE-MRI and DWI parameters were measured independently by 2 radiologists (YJZ and $\mathrm{YL}$, with 7 and 15 years of experience in gastrointestinal abdominal imaging, respectively) who were blinded to the patients' clinical and histopathological data. The region of interest (ROI) drawing principles were 
as follows: the ROI was manually traced slightly along the borders of the tumor to include the entire tumor at a single slice that showed the maximum dimension of the visible tumor, while avoiding visible blood vessels, necrotic areas, and cystic areas, on DCE-MRI and DWI, respectively.

ADC maps were created by Function Tool software on the GE ADW4.6 workstation (GE Medical Systems, USA) using DWI data. The ROI was automatically copied from the DWI images to the ADC maps, and the ADC values were obtained from the ROI on the ADC map.

Quantitative DCE-MRI analysis was performed using an inhouse-developed software, OmniKinetics 2.0.10 (Life Science, GE Healthcare, China). First, DCE images were pre-processed with three-dimensional non-rigid registration to correct the ROI displacement caused by patients' breathing and other involuntary movements. Second, the abdominal aorta was selected as the input artery on the transverse plane in the peak arterial enhancement phase. Third, the extended Tofts 2-compartment pharmacokinetic model was applied to obtain quantitative parameter maps (20), including volume transfer constant $\left(\mathrm{K}^{\text {trans }}\right)$, reverse reflux rate constant $\left(\mathrm{k}_{\mathrm{ep}}\right)$, extracellular extravascular volume fraction $\left(\mathrm{v}_{\mathrm{e}}\right)$, and plasma volume fraction $\left(\mathrm{v}_{\mathrm{p}}\right)$. By drawing a freehand ROI, $K^{\text {trans }}, k_{e p}$, $v_{e}$, and $v_{p}$ values for the selected ROI were automatically calculated.

\section{Clinical treatment}

All patients underwent D2 radical gastrectomy within 30 days after MRI and adjuvant chemotherapy with oxaliplatin and $\mathrm{S} 1(\mathrm{n}=65)$, oxaliplatin and capecitabine $(\mathrm{n}=10)$, or other $(n=4)$.

\section{Pathological examination and EMVI evaluation}

Patient records and original histopathological slides were independently re-evaluated by 2 pathologists with $>10$ years' experience in gastrointestinal pathology. The pathologists were blinded to the routine diagnoses and patient outcomes. A median of 5 paraffin-embedded tissue blocks per tumor were used for analysis. As the vessels are orientated approximately at a right angle to the gastric wall, we adopted tangential macroscopic dissection, as well as conventional perpendicular sampling, which enabled all veins from the tumor region to be thoroughly evaluated, as proposed by Dirschmid et al. and Sternberg et al. (21-23), which is routine procedure in our hospital. The pEMVI status was recorded as present or absent with hematoxylin and eosin (HE)-stained slides.

The diagnostic criteria for pEMVI adopted in the present study were based on those reported by Talbot et al. (24), the cancer datasets of the Royal College of Pathologists, United Kingdom (RCPathUK) (25), and the reporting protocol College of American Pathologists (CAP) (26). EMVI is now listed as a line item in CAP gastric cancer reporting protocols (27), and is a simple item in RCPathUK gastric cancer datasets (28). Therefore, we adopted the definition used in corresponding colorectal cancer guidelines $(25,26)$ : the presence of a rounded mass of tumor tissue within an endothelium-lined space, either surrounded by a rim of smooth muscle or containing red blood cells. Other auxiliary characteristic signs (e.g., the "protruding tongue" and the "orphan artery" signs) could indicate EMVI, according to RCPathUK and CAP guidelines $(25,26)$. To ensure consistency of the evaluation criteria, the 2 pathologists were trained with pathological diagnostic guidelines $(25,26)$ prior to the evaluation. In case of disagreement, a consensus was reached by discussion.

Pathological staging was assessed according to the 8th edition of the AJCC/UICC TNM staging system (6). The histopathological type, differentiation, Lauren's classification (29), and human epidermal growth factor receptor 2 (HER2) expression were also examined. HER2 expression was detected according to Hofmann et al.'s approach (30).

\section{Follow-up}

Follow-up after radical gastrectomy was carried out via interview in the outpatient department every 3 months for the first year, and every 6-12 months thereafter. Followup data included laboratory examinations, CT scans, and endoscopic examinations. RFS was recorded and was measured from the date of surgery to the first date of local and/or distant recurrence or the last follow-up date without recurrence. Tumor recurrence was defined as local recurrence, distant metastasis, or death caused by gastric cancer, detected by imaging or pathology. The last date of data collection was May 31, 2019.

\section{Statistical analysis}

Continuous variables with normal distribution were presented as mean \pm standard deviation, and those with abnormal distribution were expressed as medians and interquartile ranges. Categorical variables were presented as frequencies and percentages. 
Interobserver agreements for DCE-MRI parameters and $\mathrm{ADC}$ values were assessed using intraclass correlation coefficients (ICCs) with 2-way random method. The ICC was defined as follows (31): $<0.20$, poor; $0.20-0.40$, fair; $0.41-0.60$, moderate; $0.61-0.80$, good; and $\geq 0.81$, excellent. The averages of the measurements were used for further statistical analysis.

The independent samples $t$-test was used to compare normally distributed data, and the Mann-Whitney $U$-test was used to compare non-normally distributed parameters. Categorical variables were compared using the $\chi^{2}$-test or Fisher's exact test. Differences in clinicopathological features, quantitative DCE-MRI parameters, and ADC values between the pEMVI-positive and -negative groups were compared. Multivariate logistic regression was used to build the combined prediction model with the statistically significant parameters. The prediction performance of the model was evaluated by receiver operating characteristic (ROC) curve using prediction probability, quantified by the area under the curve (AUC), overall accuracy (ACC), sensitivity, specificity, negative predictive value (NPV), and positive predictive value (PPV). The optimal cutoff value for the prediction probability of the regression equation was calculated at the maximum value of the Youden index $\left(\right.$ sensitivity + specificity $\left.^{-1}\right)$. The patients were then divided into MRI-predicted EMVI (mrEMVI)-positive and -negative groups according to the cutoff value. The RFS between the 2 groups was estimated using the KaplanMeier method, and differences were compared using the log-rank test. Statistical analyses were carried out using $\mathrm{R}$ software (version 3.5.3; http://www.r-project.org/). $\mathrm{P}<0.05$ was considered to be statistically significant.

\section{Results}

\section{Clinical and pathological characteristics}

Of the 79 patients in the present study, 29 were pEMVI positive and 50 were pEMVI negative. A significant difference was observed in postoperative pathological staging between the 2 groups $(\mathrm{P}=0.015)$. No significant differences were found for sex, age, MTD, tumor site, histological type, differentiation, Lauren's classification, or HER2 expression between patients with or without pEMVI (all $\mathrm{P}>0.05$ ) (Table 1).

\section{Interobserver agreement}

The ICC values between the two radiologists for $\mathrm{K}^{\text {trans }}, \mathrm{k}_{\mathrm{ep}}$, $\mathrm{v}_{\mathrm{e}}, \mathrm{v}_{\mathrm{p}}$, and ADC were 0.925 [95\% confidence interval (CI): 0.885-0.951], 0.903 (95\% CI: 0.853-0.937), 0.877 (95\% CI: 0.814-0.919), 0.891 (95\% CI: 0.834-0.929), and 0.930 (95\% CI: 0.893-0.955), respectively, indicating almost excellent agreement.

\section{Differences in quantitative DCE-MRI parameters and $A D C$ values between pEMVI-positive and-negative groups}

Among the 79 patients, quantitative DCE-MRI parameters of $\mathrm{K}^{\text {trans }}, \mathrm{k}_{\mathrm{ep}}, \mathrm{v}_{\mathrm{e}}$, and $\mathrm{v}_{\mathrm{p}}$ were $0.107(0.058,0.176) \mathrm{min}^{-1}, 0.545$ $(0.360,0.729) \mathrm{min}^{-1}, 0.270(0.205,0.368)$, and $0.012(0.006$, $0.030)$, respectively; the ADC value was $1.29(1.21,1.58)$ $\times 10^{-3} \mathrm{~mm}^{2} / \mathrm{s}$. The comparisons of quantitative DCE-MRI parameters and ADC values between the pEMVI-positive and -negative groups are summarized in Table 2. The results showed that, in the pEMVI-positive group (Figure 2), the $\mathrm{K}^{\text {trans }}$ and $\mathrm{k}_{\mathrm{ep}}$ values were significantly higher than those in the pEMVI negative group (Figure 3), and the ADC values were significantly lower $(\mathrm{P}<0.001, \mathrm{P}<0.001$, and 0.002 , respectively), whereas $\mathrm{v}_{\mathrm{e}}$ and $\mathrm{v}_{\mathrm{p}}$ showed no significant difference $(\mathrm{P}>0.05)$.

\section{Combined prediction efficiency of pEMVI using $K^{\text {trans }}, \boldsymbol{k}_{e p}$, and $A D C$ values}

Multivariate logistic regression analysis was conducted with $\mathrm{K}^{\text {trans }}, \mathrm{k}_{\mathrm{ep}}$, and ADC values as covariables, and pEMVI as the dependent variable. The results showed that $\mathrm{K}^{\mathrm{trans}}, \mathrm{k}_{\mathrm{ep}}$, and ADC values were independently associated with the pEMVI with odds ratio (OR) values of $3.66,2.65$, and 0.30 (all $\mathrm{P}<0.05$, Table 3).

ROC analysis was performed based on the prediction probability of the regression equation (Figure 4). The cutoff value was 0.513, the AUC was 0.879 (95\% CI: 0.797-0.961), and the ACC, sensitivity, specificity, PPV, and NPV were $87.3 \%, 72.4 \%, 96.0 \%, 91.3 \%$, and $85.7 \%$, respectively. Based on the predictive results, 23 cases were found to be mrEMVI positive and 56 were mrEMVI negative.

\section{Prognostic value of mrEMVI and its association with RFS}

In the present study, the median follow-up duration for all patients was 20 months (range: 5-40 months), of which 29 of $79(36.7 \%)$ had tumor recurrence. The recurrence rate was 14 of $23(60.9 \%)$ in the mrEMVI-positive group and 15 of $56(26.8 \%)$ in the mrEMVI-negative group, with a significant difference between the 2 groups $(\mathrm{P}=0.006)$. 
Table 1 Clinical and histopathological characteristics of 79 patients in the pathological extramural venous invasion (pEMVI)-positive and -negative groups

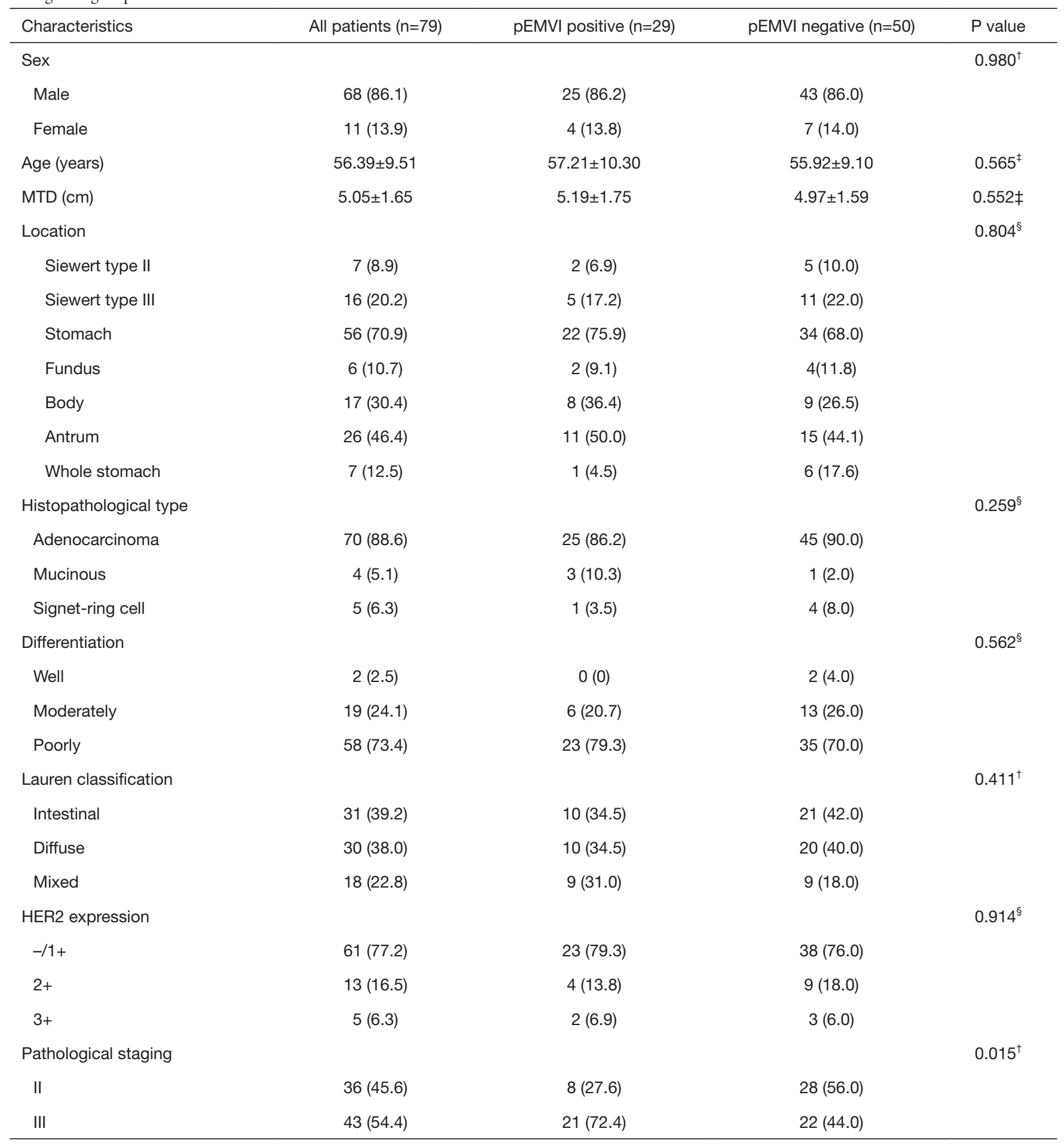

${ }^{\dagger}$ Calculated using $\chi^{2}$-test. ${ }^{\ddagger}$ Calculated using the independent samples t-test. ${ }^{\S}$ Calculated using Fisher's exact test. Continuous variables are shown as mean \pm standard deviation. Categorical variables are shown as the number of patients. with the percentage of patients in parentheses. HER2, human epidermal growth factor receptor 2; MTD, maximum tumor diameter. 
Table 2 Comparison of dynamic contrast-enhanced magnetic resonance imaging quantitative parameters and apparent diffusion coefficient (ADC) values between pathological extramural venous invasion (pEMVI)-positive and -negative groups

\begin{tabular}{|c|c|c|c|c|}
\hline Parameters & pEMVI positive $(n=29)$ & pEMVI negative $(n=50)$ & Z & $P$ value ${ }^{\dagger}$ \\
\hline $\mathrm{K}^{\mathrm{trans}}\left(\mathrm{min}^{-1}\right)$ & $0.189(0.113,0.225)$ & $0.082(0.054,0.129)$ & -3.824 & $<0.001$ \\
\hline $\mathrm{k}_{\mathrm{ep}}\left(\min ^{-1}\right)$ & $0.687(0.475,1.056)$ & $0.475(0.318,0.610)$ & -3.245 & 0.001 \\
\hline $\mathrm{v}_{\mathrm{e}}$ & $0.331(0.228,0.410)$ & $0.258(0.197,0.350)$ & -1.709 & 0.088 \\
\hline$v_{p}$ & $0.014(0.003,0.044)$ & $0.011(0.007,0.026)$ & -0.346 & 0.729 \\
\hline
\end{tabular}

${ }^{\dagger}$ Calculated using the Mann-Whitney U-test. Data are shown as medians and interquartile ranges. $\mathrm{k}_{\mathrm{ep}}$, reverse reflux rate constant; $\mathrm{K}^{\text {trans }}$, volume transfer constant; $v_{e}$, extracellular extravascular volume fraction; $v_{p}$, plasma volume fraction.

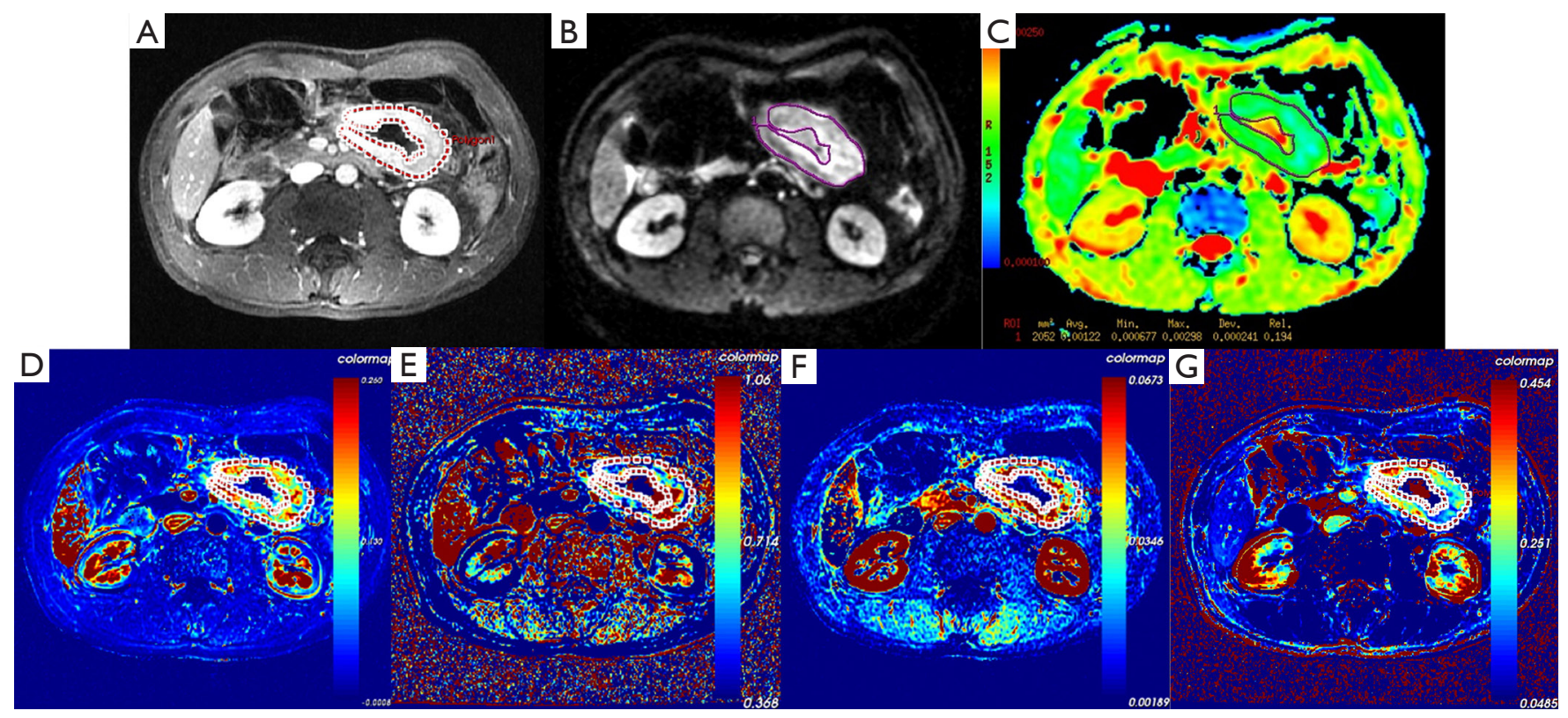

Figure 2 Images of a 39-year-old man with extramural venous invasion-positive gastric adenocarcinoma in body of the stomach. (A) In the contrast-enhanced imaging, the gastric wall was irregularly thickened and heterogeneous enhanced; (B) The lesion showed a high signal in the diffusion-weighted image. Pseudo-colorized apparent diffusion coefficient (ADC) map (C), volume transfer constant (K $\left.{ }^{\text {trans }}\right)$ map (D), reverse reflux rate constant $\left(\mathrm{k}_{\mathrm{ep}}\right)$ map $(\mathrm{E})$, extracellular extravascular volume fraction $\left(\mathrm{v}_{\mathrm{e}}\right)$ map $(\mathrm{F})$, and plasma volume fraction $\left(\mathrm{v}_{\mathrm{p}}\right)$ map $(\mathrm{G})$ shows mixed red, green, and blue in the corresponding tumor, with an ADC of $1.22 \times 10^{-3} \mathrm{~mm}^{2} / \mathrm{s}, \mathrm{K}^{\text {trans }}$ of $0.165 \mathrm{~min}^{-1}, \mathrm{k}_{\mathrm{ep}}$ of $0.911 \mathrm{~min}^{-1}$, $\mathrm{v}_{\mathrm{e}}$ of 0.231 , and $\mathrm{v}_{\mathrm{p}}$ of 0.042 .

The Kaplan-Meier survival analysis showed that the median RFS of all patients was 28.7 months (95\% CI: 25.631.8 ), and the 2 -year RFS rate was $62 \%$. The median RFS of the mrEMVI-positive group was 21.7 months (95\% CI: 18.0-25.4), and the 2-year RFS rate was $43.6 \%$. The median RFS of the mrEMVI-negative group was 31.2 months (95\% CI: 27.6-34.9), and the 2-year RFS rate was $72.5 \%$. The 2-year RFS rates were significantly associated with mrEMVI, according to the log-rank test $(\mathrm{P}=0.010)$ (Figure 5).

\section{Discussion}

In the present study, we combined DCE-MRI and DWI for the first time to build a model to predict pEMVI in LAGC and explore their prognostic significance. Our study showed that the combined prediction model based on quantitative DCE parameters and ADC values has a good prediction efficiency for pEMVI in LAGC, with an AUC value of 0.879 (95\% CI: 0.797-0.961). The RFS of the mrEMVI-positive 


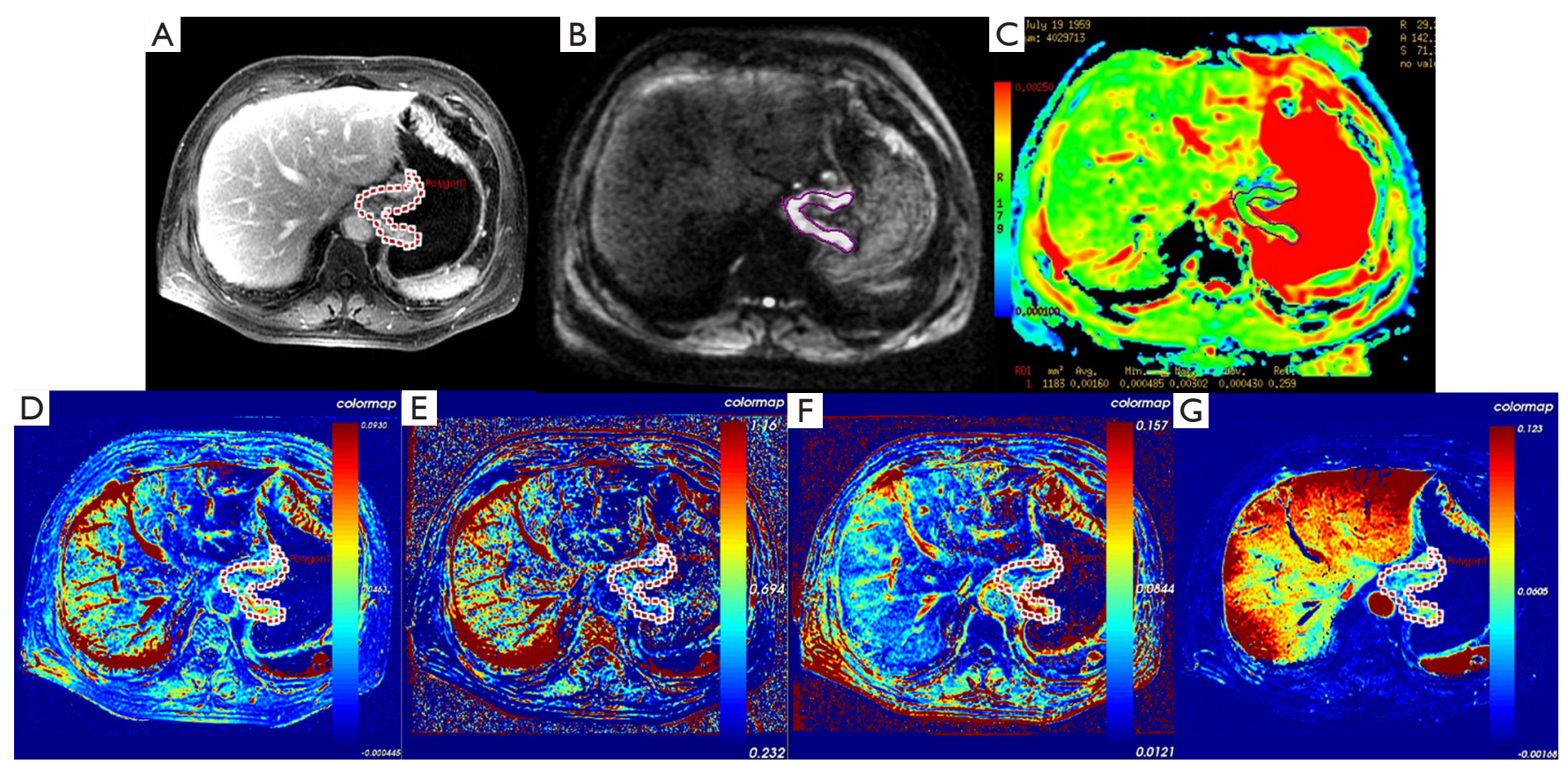

Figure 3 Images of a 58-year-old man with extramural venous invasion-negative gastric adenocarcinoma in the esophagogastric junction. (A) In the contrast-enhanced imaging, the gastric wall was irregularly thickened and heterogeneous enhancement; (B) The lesion showed a high signal in the diffusion-weighted image. Pseudo-colorized apparent diffusion coefficient (ADC) map (C), volume transfer constant $\left(\mathrm{K}^{\text {trans }}\right)$ map (D), reverse reflux rate constant $\left(\mathrm{k}_{\mathrm{ep}}\right)$ map $(\mathrm{E})$, extracellular extravascular volume fraction $\left(\mathrm{v}_{\mathrm{e}}\right)$ map $(\mathrm{F})$, and plasma volume fraction $\left(\mathrm{v}_{\mathrm{p}}\right)$ map $(\mathrm{G})$ shows mixed red, green, and blue in the corresponding tumor with an ADC of $1.60 \times 10^{-3} \mathrm{~mm}^{2} / \mathrm{s}, \mathrm{K}^{\text {trans }}$ of $0.048 \mathrm{~min}^{-1}, \mathrm{k}_{\mathrm{ep}}$ of $0.517 \mathrm{~min}^{-1}$, $\mathrm{v}_{\mathrm{e}}$ of 0.128 , and $\mathrm{v}_{\mathrm{p}}$ of 0.039 .

Table 3 Multivariate logistic regression analysis of combined dynamic contrast-enhanced magnetic resonance imaging quantitative parameters and apparent diffusion coefficient values (ADC), and predictive impact on pathological extramural venous invasion

\begin{tabular}{lccc}
\hline Parameters & Odds ratio & 95\% confidence interval & P value \\
\hline $\mathrm{K}^{\text {trans }}$ & 3.656 & $1.616-8.271$ & 0.002 \\
$\mathrm{~K}_{\mathrm{ep}}$ & 2.652 & $1.362-5.163$ & 0.004 \\
$\mathrm{ADC}$ & 0.297 & $0.126-0.701$ & 0.006 \\
(Intercept) $^{\dagger}$ & 0.450 & & 0.014 \\
\hline
\end{tabular}

"Intercept" is the constant term in the logistic regression equation. $\mathrm{k}_{\mathrm{ep}}$, reverse reflux rate constant; $\mathrm{K}^{\text {trans }}$, volume transfer constant.

group was significantly lower than that of the mrEMVInegative group.

The incidence of pathological EMVI has been reported to vary from $20 \%$ to $75 \%$ in gastric cancer due to different pathological stages and staining techniques (e.g., HE or

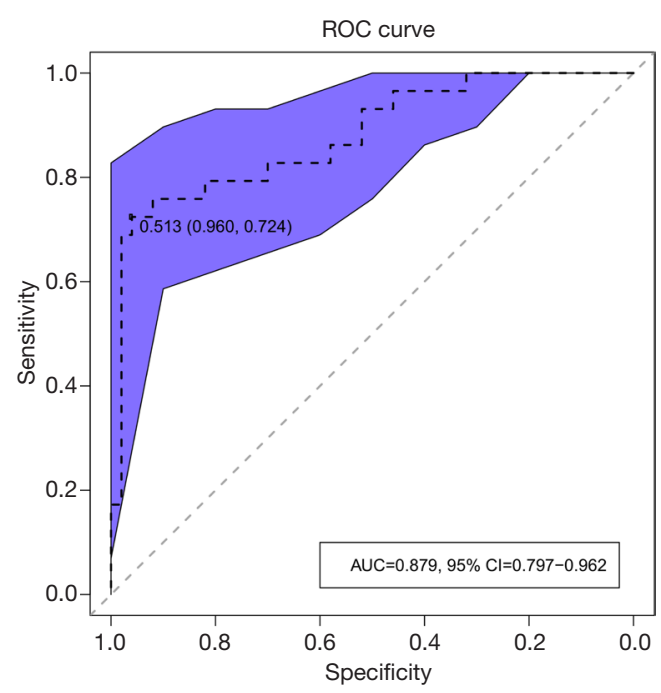

Figure 4 Receiver-operating characteristic (ROC) curve of model established by dynamic contrast-enhanced magnetic resonance imaging quantitative parameters and apparent diffusion coefficient values for the prediction of pathological extramural venous invasion in locally advanced gastric cancer. Area under the curve (AUC) was 0.879. CI, confidence interval. 


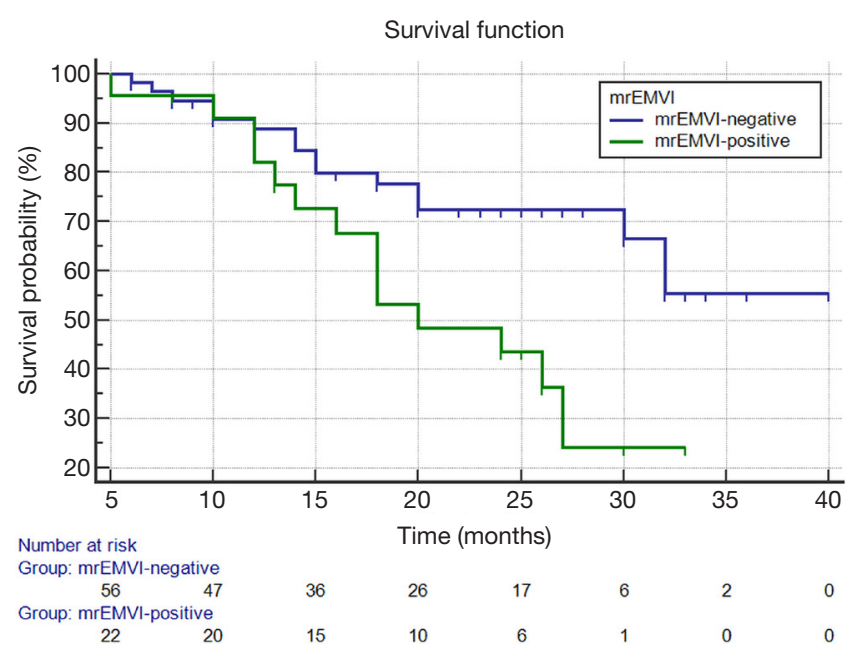

Figure 5 Kaplan-Meier recurrence-free survival (RFS) analysis of each group divided according to magnetic resonance imaging extramural venous invasion (mrEMVI). mrEMVI-positive patients had a significantly poorer RFS than patients without mrEMVI (cumulative 2 -year RFS rate: $43.6 \%$ vs. $72.5 \%, \mathrm{P}=0.010$ ).

elastic fiber staining) (11). Our study found the frequency of pEMVI was $36.7 \%$ (29/79), which was slightly lower than a recent study that reported a $51 \%$ frequency in stage III gastric cancer (32). The pathological staging of tumors is well known to be important for prognosis. In their study of 529 patients with gastric cancer, Gabbert et al. found that the incidence of EMVI increased with the depth of tumor invasion and the lymph node metastasis (9). This finding was consistent with the results of the present study; the pathological staging in the pEMVI-positive group was significantly higher than that in the pEMVI-negative group.

Traditionally, EMVI could only be detected after postoperative histopathological examination, and therefore cannot be used to predict treatment benefit. If EMVI status could be predicted preoperatively and non-invasively, appropriate and individualized treatment plans could be developed to improve the prognosis of LAGC patients. Recently, Tan et al. proposed some general CT imaging features of EMVI (11), and Kim et al. found that EMVI detected by CT was an independent prognostic factor for reduced disease-free survival in patients with gastric cancer (33). Furthermore, in a study by Cheng et al., EMVI detected by CT, combined with $\mathrm{N}$ status detected by contrast-enhanced CT, could be used as a valuable preoperative prognostic factor in patients with $\mathrm{T} 4 \mathrm{a}$ gastric cancer (34). However, these studies are all based on morphological diagnoses and depend on the experience of the radiologist. Functional MRI, such as quantitative DCEMRI and DWI, could obtain quantitative parameters, which are more objective and reproducible. In the present study, quantitative DCE-MRI and DWI were combined to predict pEMVI in LAGC, and the prognostic value of mrEMVI was explored.

In the present study, DCE-MRI parameters, $\mathrm{K}^{\text {trans }}$ and $\mathrm{k}_{\mathrm{ep}}$, in the pEMVI-positive group were significantly higher than those in the pEMVI-negative group. Previous studies of DCE-MRI in gastric cancer have focused on tumor staging, diagnosis, and the correlation of pharmacokinetic parameters with pathological prognostic factors (35-37). $\mathrm{K}^{\text {trans }}$ reflects the exchange ability of contrast agent in plasma and extracellular space, whereas $k_{\text {ep }}$ quantifies the flux rate of the contrast agent from the extracellular space back to the plasma, both of which are important markers of vascular permeability (20). Joo et al. demonstrated that $\mathrm{K}^{\text {trans }}$ was significantly correlated with the grade of epidermal growth-factor receptor expression (35). Ma et al. found that $\mathrm{K}^{\text {trans }}$ showed a significant positive correlation with vascular endothelial growth factor (36). These findings suggest that DCE-MRI reflects tumor biology, providing prognostic information for patients with gastric cancer. Yu et al. found that, in rectal cancers, $\mathrm{K}^{\text {trans }}$ and $\mathrm{k}_{\mathrm{ep}}$ values in patients with synchronous liver metastasis were significantly higher than those without metastasis (14). Li et al. reported that higher $\mathrm{K}^{\mathrm{trans}}$ and $\mathrm{k}_{\mathrm{ep}}$ values in breast cancer before neoadjuvant therapy were significantly correlated with lower diseasefree and overall survival (15). According to our findings and those of previous studies (14,15,35-37), we hypothesized that higher $\mathrm{K}^{\mathrm{trans}}$ and $\mathrm{k}_{\mathrm{ep}}$ values in the pEMVI-positive group suggest higher tumor angiogenesis permeability and blood perfusion, which could promote hematogenous metastasis, leading to the occurrence of EMVI and postoperative tumor recurrence. The extravascular extracellular space and the blood plasma volume per unit volume of tissue were reflected by $\mathrm{v}_{\mathrm{e}}$ and $\mathrm{v}_{\mathrm{p}}$, respectively. Joo et al. found that $\mathrm{v}_{\mathrm{e}}$ showed a significant positive correlation with the $\mathrm{T}$ staging of gastric cancers (35), and Ma et al. found that the diffuse type showed a higher $\mathrm{v}_{\mathrm{e}}$ than the intestinal type in the Lauren classification (36). In a previously published study, $\mathrm{v}_{\mathrm{e}}$ values were found to be significantly correlated with progression-free and overall survival at 3 years in oropharyngeal and hypopharyngeal squamous cell carcinoma (38). However, no significant differences in $\mathrm{v}_{\mathrm{e}}$ and $v_{p}$ values were found between the pEMVI-positive and -negative groups in our study. $\mathrm{v}_{\mathrm{e}}$ and $\mathrm{v}_{\mathrm{p}}$ might have more 
influence factors, and their significance in the prediction of pEMVI and prognosis of gastric cancer need to be further clarified.

DWI has been widely used in the diagnosis, treatment response, and prognostic prediction of various tumors, and the ADC value has been reported to be closely related to prognosis (16,17,39-41). In their study, Peng et al. found that quantitative whole-lesion ADC histogram analysis could help to differentiate histological grades of rectal cancer (16). Giganti et al. reported that ADC is different according to local invasion, nodal involvement, and the 7th edition TNM stage groups (39). In a recently published study with 82 patients, the ADC value of pathological lymph nodes was shown to be lower than that of benign lymph nodes (40). In their study, De Cobelli et al. reported that responders showed a significant increase in ADC compared with non-responders after neoadjuvant treatment (85.45\% increase vs. $8.21 \%$ decrease, respectively) (41). Therefore, DWI is a promising, non-invasive imaging biomarker for the evaluation of the aggressiveness of gastric cancer. In the present study, the ADC value in the pEMVI-positive group was significantly lower than that in the pEMVI-negative group, and the multivariate logistic regression results revealed that the OR value of pEMVI was 0.297, suggesting that patients with low ADC values were more likely to develop pEMVI. The ADC value reflects the diffusion of water molecules in tumor tissues. A lower ADC value indirectly indicates increased density, proliferation activity, and atypia of tumor cells, along with imbalance of the cytoplasmic ratio and reduced extracellular space. Tumor cells in tumor tissues with low ADC values are more likely to migrate into vessels, and eventually cause tumor recurrence and metastasis, than those with high ADC values.

In the present study, we attempted to combine $\mathrm{K}^{\text {trans }}$, $\mathrm{k}_{\mathrm{ep}}$, and $\mathrm{ADC}$ values to predict the occurrence of $\mathrm{pEMVI}$ in LAGC for the first time. ROC analysis showed that combined quantitative DCE and DWI multiparameters resulted in good prediction performance in terms of pEMVI, with an AUC of 0.879 , and sensitivity and specificity of $72.4 \%$ and $96 \%$, respectively. To further investigate the relationship between mrEMVI and patient prognosis, Kaplan-Meier survival analysis was performed on the 79 patients. The results showed that the tumor recurrence rate in the mrEMVI-positive group was significantly higher than in the mrEMVI-negative group ( $60.9 \%$ vs. $26.8 \%$ ), and the RFS in the mrEMVI-positive group was significantly lower than that in the mrEMVI- negative group (21.9 vs. 31.2 months). Nishibeppu et al. found that the incidence of hematogenous metastasis in pEMVI-positive stage III gastric cancer patients was significantly higher than that in the negative group (32). This may be attributed to tumor cells derived from the primary lesion entering the blood circulation, resulting in distant organ metastasis. mrEMVI makes it possible to predict pEMVI preoperatively, which is an important stratified risk factor and can provide a basis for the selection of an individualized treatment plan for LAGC patients. Because tumor cells can exist in the blood circulation before surgery, for patients with a high risk of EMVI or those who are mrEMVI positive, neoadjuvant therapy, intensive postoperative adjuvant therapy, or the addition of antiangiogenic molecular-targeted drugs (i.e., trastuzumab) may be needed in clinical practice.

The present study has some limitations. First, the sample size was relatively small, and more cases are needed for validation. Second, the patients enrolled in the study were diagnosed with LAGC, and early gastric cancer patients were not included; therefore, selection bias was unavoidable. Third, the manual drawing of the ROI may have introduced a certain amount of subjectivity. Fourth, pEMVI was detected on routine HE staining slides only, and not with elastin staining. Special staining for elastin fiber could help to identify venous invasion, but itis not routinely performed for all patients in our hospital. However, special staining is not recommended in the current practice guidelines, and these techniques are also labor intensive, time consuming, and expensive. Despite this, we took some measures to improve the rate of EMVI detection, such as tangential sampling, unifying assessment criteria, training the pathologists with pathological diagnostic guidelines $(25,26)$ before evaluation, and using auxiliary signs to aid detection. These measures might have offset the reduced detection rate caused by the absence of elastin staining to some extent. Finally, due to the short follow-up time (median follow-up time, 20 months), no analysis of overall survival was conducted.

\section{Conclusions}

The preoperative quantitative DCE-MRI parameters $\mathrm{K}^{\text {trans }}$ and $\mathrm{k}_{\mathrm{ep}}$, and the DWI parameter $\mathrm{ADC}$, are independent predictors of pEMVI in LAGC. Also, mrEMVI based on the logistic regression model helped to predict the RFS of patients. This is of great value to clinicians when choosing the most appropriate treatment strategy for patients. 


\section{Acknowledgments}

Funding: This work was supported by the Beijing Hope Run Special Fund (no. LC2016A06).

\section{Footnote}

Conflicts of Interest: All authors have completed the ICMJE uniform disclosure form (available at http://dx.doi. org/10.21037/qims-20-246). The authors have no conflicts of interest to declare.

Ethical Statement: The present study was approved by the Independent Ethics Committee of the Cancer Hospital, Chinese Academy of Medical Sciences (Beijing, China), and the requirement for informed patient consent was waived (no. 2019081617374702).

Open Access Statement: This is an Open Access article distributed in accordance with the Creative Commons Attribution-NonCommercial-NoDerivs 4.0 International License (CC BY-NC-ND 4.0), which permits the noncommercial replication and distribution of the article with the strict proviso that no changes or edits are made and the original work is properly cited (including links to both the formal publication through the relevant DOI and the license). See: https://creativecommons.org/licenses/by-nc-nd/4.0/.

\section{References}

1. Torre LA, Bray F, Siegel RL, Ferlay J, Lortet-Tieulent J, Jemal A. Global cancer statistics. CA Cancer J Clin 2015;65:87-108.

2. Jemal A, Siegel R, Xu J, Ward E. Cancer statistics, 2010. CA Cancer J Clin 2010;60:277-300.

3. Cuschieri A, Weeden S, Fielding J, Bancewicz J, Craven J, Joypaul V, Sydes M, Fayers P. Patient survival after D1 and D2 resections for gastric cancer: long-term results of the MRC randomized surgical trial. Surgical Co-operative Group. Br J Cancer 1999;79:1522-30.

4. Fitzgerald TL, Efird JT, Bellamy N, Russo SM, Jindal C, Mosquera C, Holliday EG, Biswas T. Perioperative chemotherapy versus postoperative chemoradiotherapy in patients with resectable gastric/gastroesophageal junction adenocarcinomas: A survival analysis of 5058 patients. Cancer 2017;123:2909-17.

5. Schuhmacher C, Gretschel S, Lordick F, Reichardt P, Hohenberger W, Eisenberger CF, Haag C, Mauer
ME, Hasan B, Welch J, Ott K, Hoelscher A, Schneider PM, Bechstein W, Wilke H, Lutz MP, Nordlinger B, Van Cutsem E, Siewert JR, Schlag PM. Neoadjuvant chemotherapy compared with surgery alone for locally advanced cancer of the stomach and cardia: European Organisation for Research and Treatment of Cancer randomized trial 40954. J Clin Oncol 2010;28:5210-8.

6. Amin MB, Edge SB, Greene FL, et al. AJCC cancer staging manual, 8th edn. Springer, Chicago, 2017.

7. Li P, He HQ, Zhu CM, Ling YH, Hu WM, Zhang XK, Luo RZ, Yun JP, Xie D, Li YF, Cai MY. The prognostic significance of lymphovascular invasion in patients with resectable gastric cancer: a large retrospective study from Southern China. BMC Cancer 2015;15:370.

8. Messenger DE, Driman DK, Kirsch R. Developments in the assessment of venous invasion in colorectal cancer: implications for future practice and patient outcome. Hum Pathol 2012;43:965-73.

9. Gabbert HE, Meier S, Gerharz CD, Hommel G. Incidence and prognostic significance of vascular invasion in 529 gastric-cancer patients. Int J Cancer 1991;49:203-7.

10. Streutker CJ. Extramural Venous Invasion in Patients with Locally Advanced Esophageal Cancer: A Reminder to Pathologists to Look Harder. Ann Surg Oncol 2018;25:1465-6.

11. Tan CH, Vikram R, Boonsirikamchai P, Bhosale P, Marcal L, Faria S, Charnsangavej C. Extramural venous invasion by gastrointestinal malignancies: CT appearances. Abdom Imaging 2011;36:491-502.

12. Wáng YXJ, Wang X, Wu P, Wang Y, Chen W, Chen H, Li $\mathrm{J}$. Topics on quantitative liver magnetic resonance imaging. Quant Imaging Med Surg 2019;9:1840-90.

13. García-Figueiras R, Padhani AR, Beer AJ, BaleatoGonzález S, Vilanova JC, Luna A, Oleaga L, GómezCaamaño A, Koh DM. Imaging of Tumor Angiogenesis for Radiologists--Part 1: Biological and Technical Basis. Curr Probl Diagn Radiol 2015;44:407-24.

14. Yu J, Xu Q, Huang DY, Song JC, Li Y, Xu LL, Shi HB. Prognostic aspects of dynamic contrast-enhanced magnetic resonance imaging in synchronous distant metastatic rectal cancer. Eur Radiol 2017;27:1840-7.

15. Li SP, Makris A, Beresford MJ, Taylor NJ, Ah-See ML, Stirling JJ, d'Arcy JA, Collins DJ, Kozarski R, Padhani AR. Use of dynamic contrast-enhanced MR imaging to predict survival in patients with primary breast cancer undergoing neoadjuvant chemotherapy. Radiology 2011;260:68-78.

16. Peng Y, Tang H, Meng X, Shen Y, Hu D, Kamel I, Li Z. Histological grades of rectal cancer: whole-volume 
histogram analysis of apparent diffusion coefficient based on reduced field-of-view diffusion-weighted imaging. Quant Imaging Med Surg 2020;10:243-56.

17. Giganti F, Orsenigo E, Esposito A, Chiari D, Salerno A, Ambrosi A, Albarello L, Mazza E, Staudacher C, Del Maschio A, De Cobelli F. Prognostic Role of Diffusionweighted MR Imaging for Resectable Gastric Cancer. Radiology 2015;276:444-52.

18. National Comprehensive Cancer Network. NCCN clinical practice guidelines in oncology (NCCN Guidelines $\left.{ }^{\circledR}\right)$ : Gastric Cancer, Version 2.2020. Available online: https:// www.nccn.org/professionals/physician_gls/pdf/gastric.pdf (accessed 13 May 2020).

19. Tao X, Wang L, Hui Z, Liu L, Ye F, Song Y, Tang Y, Men Y, Lambrou T, Su Z, Xu X, Ouyang H, Wu N. DCE-MRI Perfusion and Permeability Parameters as predictors of tumor response to CCRT in Patients with locally advanced NSCLC. Sci Rep 2016;6:35569.

20. Tofts PS, Brix G, Buckley DL, Evelhoch JL, Henderson E, Knopp MV, Larsson HB, Lee TY, Mayr NA, Parker GJ, Port RE, Taylor J, Weisskoff RM. Estimating kinetic parameters from dynamic contrast-enhanced T(1)-weighted MRI of a diffusable tracer: standardized quantities and symbols. J Magn Reson Imaging 1999;10:223-32.

21. Dirschmid K, Sterlacci W, Oellig F, Edlinger M, Jasarevic Z, Rhomberg M, Dirschmid H, Offner F. Absence of extramural venous invasion is an excellent predictor of metastasis-free survival in colorectal carcinoma stage II-a study using tangential tissue sectioning. J Clin Pathol 2012;65:619-23.

22. Dirschmid K, Sterlacci W, Wöll E, Tschann P, Rhomberg $M$, Offner F. Incidence of extramural venous invasion in colorectal carcinoma as determined at the invasive tumor front and its prognostic impact. Hum Pathol 2019;86:102-7.

23. Sternberg A, Mizrahi A, Amar M, Groisman G. Detection of venous invasion in surgical specimens of colorectal carcinoma: the efficacy of various types of tissue blocks. J Clin Pathol 2006;59:207-10.

24. Talbot IC, Ritchie S, Leighton MH, Hughes AO, Bussey $\mathrm{HJ}$, Morson BC. The clinical significance of invasion of veins by rectal cancer. Br J Surg 1980;67:439-42.

25. Maurice B Loughrey, Philip Quirke, Neil A Shepherd. Dataset for histopathological reporting of colorectal cancer (4th edition). Available online: https://www.rcpath. org/resourceLibrary/g049-dataset-for-histopathologicalreporting-of-colorectal-cancer.html (accessed Sep 2018).

26. Lawrence J. Burgart, Sanjay Kakar, Chanjuan Shi,
Mariana E. Berho, David K. Driman, MBchB, Patrick Fitzgibbons, Wendy L. Frankel, Kalisha A. Hill, John Jessup, Alyssa M. Krasinskas, Mary K Washington.

Protocol for the examination of resection specimens from patients with primary carcinoma of the colon and rectum (v4.1.0.0). Available online: https://documents.cap.org/ protocols/cp-gilower-colonrectum-resection-20-4100.pdf (accessed Feb 2020).

27. Chanjuan Shi, Jordan Berlin, Philip A. Branton, Patrick L. Fitzgibbons, Wendy L. Frankel, Wayne L. Hofstetter, Sanjay Kakar, David Kelsen, Veronica Klepeis, Jason Talmadge Lewis, Laura H. Tan, Mary K. Washington. Protocol for the Examination of Specimens From Patients With Carcinoma of the Stomach (v4.1.0.0). Available online: https://documents.cap.org/protocols/cp-giupperstomach-20-4100.pdf (accessed Feb 2020).

28. Marco R Novelli. Dataset for the histopathological reporting of gastric carcinoma (2nd edition). Available online: https://www.rcpath.org/resourceLibrary/datasetfor-the-histopathological-reporting-of-gastric-carcinoma. html(accessed January 2007).

29. Lauren P. The two histologic main types of gastric carcinoma: diffuse and so-called intestinal type carcinoma, an attempt at a histo-clinical classification. Acta Pathol Microbiol Scand 1965;64:31-49.

30. Hofmann M, Stoss O, Shi D, Büttner R, van de Vijver M, Kim W, Ochiai A, Rüschoff J, Henkel T. Assessment of a HER2 scoring system for gastric cancer: results from a validation study. Histopathology 2008;52:797-805.

31. Landis JR, Koch GG . The measurement of observer agreement for categorical data. Biometrics 1977;33:159-74.

32. Nishibeppu K, Komatsu S, Ichikawa D, Imamura T, Kosuga T, Okamoto K, Konishi H, Shiozaki A, Fujiwara $\mathrm{H}$, Otsuji E. Venous invasion as a risk factor for recurrence after gastrectomy followed by chemotherapy for stage III gastric cancer. BMC Cancer 2018;18:108.

33. Kim TU, Kim S, Lee NK, Kim HJ, Han GJ, Lee JW, Baek HJ, Jeon TY, Kim HS, Park DY. Prognostic Value of Computed Tomography-Detected Extramural Venous Invasion to Predict Disease-Free Survival in Patients With Gastric Cancer. J Comput Assist Tomogr 2017;41:430-6.

34. Cheng J, Feng C, Zhang Y, Hong N, Ye Y, Wang Y. CT-Detected Extramural Vessel Invasion and Regional Lymph Node Involvement in Stage T4a Gastric Cancer for Predicting Progression-Free Survival. AJR Am J Roentgenol 2019;212:1-7.

35. Joo I, Lee JM, Han JK, Yang HK, Lee HJ, Choi BI. Dynamic contrast-enhanced MRI of gastric 
cancer: Correlation of the perfusion parameters with pathological prognostic factors. J Magn Reson Imaging 2015;41:1608-14.

36. Ma L, Xu X, Zhang M, Zheng S, Zhang B, Zhang W, Wang P. Dynamic contrast-enhanced MRI of gastric cancer: Correlations of the pharmacokinetic parameters with histological type, Lauren classification, and angiogenesis. Magn Reson Imaging 2017;37:27-32.

37. Li HH, Zhu H, Yue L, Fu Y, Grimm R, Stemmer A, Fu CX, Peng WJ. Feasibility of free-breathing dynamic contrast-enhanced MRI of gastric cancer using a goldenangle radial stack-of-stars VIBE sequence: comparison with the conventional contrast-enhanced breath-hold 3D VIBE sequence. Eur Radiol 2018;28:1891-9.

38. Ng SH, Liao CT, Lin CY, Chan SC, Lin YC, Yen TC, Chang JT, Ko SF, Fan KH, Wang HM, Yang LY, Wang JJ. Dynamic contrast-enhanced MRI, diffusion-weighted MRI and 18F-FDG PET/CT for the prediction of survival in oropharyngeal or hypopharyngeal squamous

Cite this article as: Zhu Y, Zhou Y, Zhang W, Xue L, Li Y, Jiang J, Zhong Y, Wang S, Jiang L. Value of quantitative dynamic contrast-enhanced and diffusion-weighted magnetic resonance imaging in predicting extramural venous invasion in locally advanced gastric cancer and prognostic significance. Quant Imaging Med Surg 2021;11(1):328-340. doi: 10.21037/ qims-20-246 cell carcinoma treated with chemoradiation. Eur Radiol 2016;26:4162-72.

39. Giganti F, Ambrosi A, Chiari D, Orsenigo E, Esposito A, Mazza E, Albarello L, Staudacher C, Del Maschio A, De Cobelli F. Apparent diffusion coefficient by diffusionweighted magnetic resonance imaging as a sole biomarker for staging and prognosis of gastric cancer. Chin J Cancer Res 2017;29:118-26.

40. Zhong J, Zhao W, Ren F, Qi S, Wang X, Lv T, Su Z, Yin H, Ren J, Huan Y. Lymph node metastasis in patients with gastric cancer: a multi-modality, morphologic and functional imaging study. Am J Transl Res 2016;8:5601-9.

41. De Cobelli F, Giganti F, Orsenigo E, Cellina M, Esposito A, Agostini G, Albarello L, Mazza E, Ambrosi A, Socci C, Staudacher C, Del Maschio A. Apparent diffusion coefficient modifications in assessing gastro-oesophageal cancer response to neoadjuvant treatment: comparison with tumour regression grade at histology. Eur Radiol 2013;23:2165-74. 